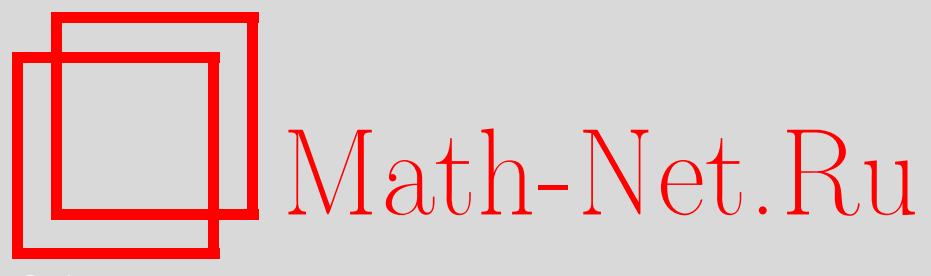

Г. С. Дерябина, А. Н. Красильников, Неконечнобазируемое многообразие центрально-метабелевых групп с отмеченной точкой, Матем. заметки, 2014, том 95, выпуск $5,795-797$

DOI: https://doi.org/10.4213/mzm10122

Использование Общероссийского математического портала Math-Net.Ru подразумевает, что вы прочитали и согласны с пользовательским соглашением http://www . mathnet.ru/rus/agreement

Параметры загрузки:

IP: 54.224 .60 .19

26 апреля 2023 г., 13:12:25

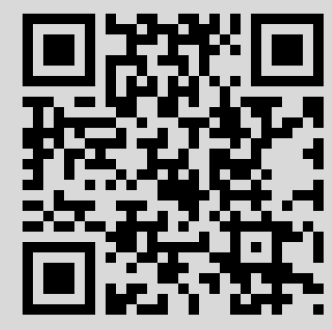




\section{Неконечнобазируемое многообразие центрально-метабелевых групп с отмеченной точкой}

\section{Г. С. Дерябина, А. Н. Красильников}

Группой с отмеченной точкой (pointed-group) называется пара $(G, g)$, состоящая из группы $G$ и выделенного элемента $g \in G$. Группы с отмеченной точкой - это группы с одной дополнительной нульарной операцией; они является алгебрами в смысле универсальной алгебры, а класс всех групп с отмеченной точкой является многообразием алгебр (определения и основные факты, связанные с тождествами и многообразиями алгебр, см., например, в [1]). О подмногообразиях этого многообразия говорят как о многообразиях групп с отмеченной точкой. Тождества и многообразия групп с отмеченной точкой впервые начал изучать Брайнт [2].

Пусть $F$ - свободная группа со свободными порождающими $y, x_{1}, x_{2}, \ldots$. Нетрудно видеть, что $(F, y)$ - свободный объект в классе всех групп с отмеченной точкой. Элемент $v=v\left(y, x_{1}, \ldots, x_{n}\right) \in F$ является тождеством группы $G$ с отмеченной точкой $g \in G$, если $v\left(g, h_{1}, \ldots, h_{n}\right)=1$ для любых $h_{1}, \ldots, h_{n} \in G$. Многообразие групп с отмеченной точкой это класс всех групп с отмеченной точкой, удовлетворяющих заданному множеству тождеств $\left\{v_{j} \mid j \in \mathbb{N}\right\}$. Напомним, что многообразие конечно базируемо, если оно может быть задано конечным множеством тождеств.

Многообразия групп с отмеченной точкой в одних ситуациях "ведут себя" так же, как многообразия "обычных" групп, а в других ситуациях "поведение" этих многообразий отличается. Существуют конечные группы с отмеченной точкой, тождества которых не допускают конечного базиса [2], тогда как тождества “обычной” конечной группы всегда имеют такой базис [3]. С другой стороны, каждое многообразие "обычных" групп, являющихся нильпотентными, метабелевыми или расширениями нильпотентной группы с помощью абелевой конечной экспоненты, конечно базируемо [4]-[6], и это же справедливо для многообразий групп с отмеченной точкой, которые нильпотентны, метабелевы [7], [8] или являются расширениями нильпотентной группы с помощью абелевой конечной экспоненты [9].

Цель нашей заметки - показать, что в центрально-метабелевом случае многообразия групп с отмеченной точкой ведут себя не так, как многообразия "обычных" групп. Напомним, что группа $G$ иентрально-метабелева, если она удовлетворяет тождеству

$$
\left[\left[\left[x_{1}, x_{2}\right],\left[x_{3}, x_{4}\right]\right], x_{5}\right]
$$

или, эквивалентно, если факторгруппа $G / Z(G)$ группы $G$ по центру $Z(G)$ метабелева. Любое многообразие "обычных" центрально-метабелевых групп конечнобазируемо [10], как и любое многообразие групп с нильпотентным коммутантом [11].

Теорема 1. Существует не конечно базируемое многообразие центрально-метабелевых групп с отмеченной точкой.

Эта теорема дает положительный ответ на вопрос Квика [9; с. 615] о существовании не конечно базируемого многообразия групп с отмеченной точкой, группы которого содержатся в наследственно конечно базируемом многообразии групп. Напомним, что многообразие наследственно конечно базируемо, если все его подмногообразия, включая само многообразие, конечно базируемы; наследственно конечно базируемым является, в частности, многообразие всех "обычных" центрально-метабелевых групп [10].

Теорема 1 сразу вытекает из следующего результата.

Работа выполнена при поддержке Российского фонда фундаментальных исследований (грант № 11-01-00945) и гранта CNPq-FAPDF PRONEX 2009/00091-0 (193.000.580/2009), Бразилия.

DOI: $10.4213 / \mathrm{mzm} 10122$ 
Теорема 2. Пусть $\mathbf{V}$ - многообразие групп с отмеченной точкой, заданное тождествами

$$
\left[\left[\left[x_{1}, x_{2}\right],\left[x_{3}, x_{4}\right]\right], x_{5}\right] \quad u \quad\left\{\left[y^{x_{1}^{p}}, y\right] \mid p \in \mathbb{P}\right\},
$$

где $\mathbb{P}$ - множество всех простых чисел. Тогда $\mathbf{V}$ не является конечно базируемым многообразием групп с отмеченной точкой.

ДокАЗАТЕЛЬСтво тЕОРЕмы 2. Пусть $w_{p}=\left[y^{x_{1}^{p}}, y\right]$. Мы докажем, что для любого простого $p$ тождество $w_{p}$ не является следствием множества тождеств, состоящего из тождества $\left[\left[\left[x_{1}, x_{2}\right],\left[x_{3}, x_{4}\right]\right], x_{5}\right]$ и тождеств $w_{q}$ для всех простых $q \neq p$. Для этого мы предъявим для каждого простого $p$ центрально-метабелеву группу $H_{p}$ с отмеченной точкой $b_{p}$, которая удовлетворяет всем тождествам $w_{q}(q \neq p)$, но не удовлетворяет тождеству $w_{p}$.

Пусть $C=\langle t\rangle_{\infty}-$ бесконечная циклическая группа, порожденная элементом $t, \mathbb{F}_{2}$ - поле из двух элементов, а $\mathbb{F}_{2} C$ - групповая алгебра группы $C$ над $\mathbb{F}_{2}$. Пусть

$$
H=\left(\begin{array}{ccc}
1 & \mathbb{F}_{2} C & \mathbb{F}_{2} C \\
0 & C & \mathbb{F}_{2} C \\
0 & 0 & 1
\end{array}\right)=\left\{\left(\begin{array}{ccc}
1 & f_{12} & f_{13} \\
0 & c & f_{23} \\
0 & 0 & 1
\end{array}\right) \mid c \in C, f_{i j} \in \mathbb{F}_{2} C\right\}
$$

- мультипликативная группа матриц. Мы будем отождествлять группу $C$ с подгруппой всех диагональных матриц группы $H$,

$$
C=\left\{\left(\begin{array}{lll}
1 & 0 & 0 \\
0 & c & 0 \\
0 & 0 & 1
\end{array}\right) \mid c \in C\right\}
$$

Пусть $N$ - подгруппа группы $H$, состоящая из матриц с единичной диагональю,

$$
N=\left\{\left(\begin{array}{ccc}
1 & f_{12} & f_{13} \\
0 & 1 & f_{23} \\
0 & 0 & 1
\end{array}\right) \mid f_{i j} \in \mathbb{F}_{2} C\right\} .
$$

Ясно, что $H$ - полупрямое произведение $C$ и $N, H=C \ltimes N$. Каждый элемент $h$ группы $H$ представим в виде $h=h_{1} c$, где $h_{1} \in N, c \in C$.

Отметим, что группа $H$ центрально-метабелева. Действительно, $H^{\prime} \subseteq N$, поэтому каждый элемент из $H^{\prime \prime}$ имеет вид

$$
\left(\begin{array}{ccc}
1 & 0 & f_{13} \\
0 & 1 & 0 \\
0 & 0 & 1
\end{array}\right), \quad f_{13} \in \mathbb{F}_{2} C,
$$

а все элементы такого вида центральны в $H$.

Пусть $p$ - произвольное, но фиксированное простое число. Пусть $V_{p}$ - линейное подпространство в $\mathbb{F}_{2} C$, порожденное всеми элементами $t^{n}$ такими, что $n \neq \pm p$. Пусть $N_{p}-$ множество всех матриц вида (1) таких, что $f_{13} \in V_{p}$. Легко видеть, что $N_{p}$ - подгруппа в $H$, лежащая в центре $Z(H)$.

Пусть $H_{p}=H / N_{p}$. Пусть

$$
b_{p}=b N_{p}, \quad \text { где } \quad b=\left(\begin{array}{lll}
1 & 1 & 0 \\
0 & 1 & 1 \\
0 & 0 & 1
\end{array}\right) .
$$

Ясно, что $H_{p}$ центрально-метабелева. Проверим, что группа с отмеченной точкой $\left(H_{p}, b_{p}\right)$ не удовлетворяет тождеству $w_{p}$, но удовлетворяет всем тождествам $w_{q}$ при $q \neq p$.

Лемма 1. Пусть $h=h_{1} c$, где $h_{1} \in N, c \in C$. Пусть $k$ - положительное целое число. Тогда

$$
\left[b^{h^{k}}, b\right]=\left[b^{c^{k}}, b\right] .
$$


ДоказАтельство. Ясно, что $h^{k}=h^{\prime} c^{k}$, где $h^{\prime} \in N$. Отметим, что $b^{h^{\prime}}=b\left[b, h^{\prime}\right]$, где $\left[b, h^{\prime}\right] \in Z(M)$. Получаем

$$
\left[b^{h^{k}}, b\right]=\left[\left(b^{h^{\prime}}\right)^{c^{k}}, b\right]=\left[\left(b\left[b, h^{\prime}\right]\right)^{c^{k}}, b\right]=\left[b^{c^{k}}\left[b, h^{\prime}\right], b\right]=\left[b^{c^{k}}, b\right]
$$

как и требовалось.

Хорошо известна и легко проверяется

Лемма 2. Пусть

$$
u=\left(\begin{array}{ccc}
0 & u_{12} & u_{13} \\
0 & 0 & u_{23} \\
0 & 0 & 0
\end{array}\right), \quad v=\left(\begin{array}{ccc}
0 & v_{12} & v_{13} \\
0 & 0 & v_{23} \\
0 & 0 & 0
\end{array}\right) .
$$

Тогда

$$
(1+u)^{-1}(1+v)^{-1}(1+u)(1+v)=1+(u v-v u) .
$$

Из леммы 2 немедленно вытекает, что $(1+u)^{-1}(1+v)^{-1}(1+u)(1+v)$ - матрица вида $(1)$, в которой $f_{13}=u_{12} v_{23}-u_{23} v_{12}$.

Пусть $h$ - произвольный элемент группы $H$. Тогда $h=h_{1} t^{n}$, где $h_{1} \in N, n \in \mathbb{Z}$. Так как

$$
b^{t^{l}}=\left(\begin{array}{ccc}
1 & t^{l} & 0 \\
0 & 1 & t^{-l} \\
0 & 0 & 1
\end{array}\right) \quad \text { для любого } l \in \mathbb{Z},
$$

то из леммы 1 и замечания к лемме 2 следует, что $\left[b^{h^{k}}, b\right]$ - матрица вида $(1)$, в которой $f_{13}=t^{k n}-t^{-k n}$. Если $k=q-$ простое число, $q \neq p$, то $f_{13}=t^{q n}-t^{-q n} \in V_{p}$, поэтому $\left[b^{h^{q}}, b\right] \in$ $N_{p}$. Это означает, что группа с отмеченной точкой $\left(H_{p}, b_{p}\right)$ удовлетворяет тождеству $w_{q}$, если $q \neq p$. С другой стороны, если $k=p$ и $h=t$, то $f_{13}=t^{p}-t^{-p} \notin V_{p}$, поэтому $\left[b^{t^{p}}, b\right] \notin$ $N_{p}$, т.е. $\left(H_{p}, b_{p}\right)$ не удовлетворяет тождеству $w_{p}$. Теорема 2 доказана.

Отметим, что наряду с тождествами групп с отмеченной точкой можно изучать тождества ассоциативных колец с отмеченной точкой. Примеры ассоциативных колец без конечного базиса тождеств были найдены Беловым [12], [13], Гришиным [14] и Щиголевым [15], обзор результатов, связанных с проблемами типа Шпехта, можно найти, например, в [16].

\section{СПИСОК ЦИТИРОВАННОЙ ЛИТЕРАТУРЫ}

[1] Ю. А. Бахтурин, А. Ю. Ольшанский, Алгебра - 2, Итоги науки и техн. Сер. Соврем. пробл. мат. Фундам. направления, 18, ВИНИТИ, М., 1988, 117-240. [2] R. M. Bryant, Bull. London Math. Soc., 14 (1982), 119-123. [3] S. Oates, M. B. Powell, J. Algebra, 1:1 (1964), 11-39. [4] D. E. Cohen, J. Algebra, 5:3 (1967), 267-273. [5] А. Н. Красильников, А. Л. Шмелькин, Алгебра и логика, 20 (1981), 546-554. [6] R. C. Lyndon, Proc. Amer. Math. Soc., 3 (1952), 579-583. [7] Z. Ali, A. Majeed, Ann. Univ. Sci. Budapest. Eötvös Sect. Math., 38 (1995), 3-11. [8] Z. Ali, A. Majeed, Bull. Korean Math. Soc., 35:4 (1998), 777-783. [9] M. Quick, Arch. Math. (Basel), 81:6 (2003), 614-620. [10] S. McKay, Proc. London Math. Soc. (3), 24 (1972), 243-256. [11] А. Н. Красильников, Изв. АН СССР. Сер. матем., 54:6 (1990), 1181-1195. [12] А. Я. Белов, Фундамент. и прикл. матем., 5:1 (1999), 47-66. [13] А. Я. Белов, Матем. сб., 191:3 (2000), 13-24. [14] А. В. Гришин, Фундамент. и прикл. матем., 5:1 (1999), 101-118. [15] В. В. Щиголев, Фундамент. и прикл. матем., 5:1 (1999), 307-312. [16] А. Я. Белов, Изв. РАН. Сер. матем., 74:1 (2010), 3-134.

\section{Г. С. Дерябина}

Поступило

Московский государственный

24.08 .2012

технический университет им. Н. Э. Баумана

E-mail: galina_deryabina@mail.ru

Исправленный вариант

26.09.2013

\section{А. Н. Красильников}

University of Brasilia, Бразилия

E-mail: alexei@unb.br 